\title{
Significance of DNA methyltransferase-1 and histone deacetylase- 1 in pancreatic cancer
}

\author{
WEI WANG ${ }^{*}$, JUN GAO* ${ }^{*}$ XIAO-HUA MAN, ZHAO-SHEN LI and YAN-FANG GONG \\ Department of Gastroenterology, Changhai Hospital, Second Military Medical University, Shanghai, P.R. China
}

Received February 2, 2009; Accepted March 13, 2009

DOI: $10.3892 /$ or 00000372

\begin{abstract}
Epigenetic modifications play an important role during carcinogenesis. The main goal of this study was to examine expression levels of two critical enzymes, DNA methyltransferase-1 (DNMT1) and histone deacetylase-1 (HDAC1), by immunohistochemistry (IHC) in human pancreatic cancer and precancerous lesions: 20 foci containing normal ductal epithelial cells without an inflammatory background (DE), 30 containing ductal epithelial cells with an inflammatory background (DEI), 48 of pancreatic intraepithelial neoplasia-1A (PanIN-1A), 103 of PanIN-1B, 99 of PanIN-2, 30 of PanIN-3, 18 of intraductal papillary mucinous neoplasm A (IPMA), 10 of IPMB, 20 of IPMC, and 54 of pancreatic ductal adenocarcinoma (PDAC). The expression levels of both DNMT1 and HDAC1 increased from normal to precancerous lesions to pancreatic cancer, in a malignancydependent manner. Correlations between expression levels and clinicopathological features of the 54 PDAC patients were also analyzed. The expression of DNMT1 significantly correlated with nerve infiltration, degree of tumor differentiation and TNM staging $(\mathrm{p}<0.05)$, while that of HDAC1 correlated with proliferative activity, degree of tumor differentiation and TNM staging $(\mathrm{p}<0.05)$. Patients with higher expression of DNMT1 and/or HDAC1 had an overall lower survival than those with lower expression $(p<0.05)$. Higher expression of DNMT1 and HDAC1 correlated with advanced stages of the disease and reflect the malignancy of pancreatic carcinoma. They may become new prognostic markers and potential therapeutic targets for pancreatic cancer.
\end{abstract}

Correspondence to: Dr Zhao-Shen Li, Department of Gastroenterology, Second Military Medical University, Shanghai, 200433, P.R. China

E-mail: lizhaoshen112@yahoo.cn

${ }^{*}$ Contributed equally

Key words: pancreatic intraepithelial neoplasia, intraductal papillary mucinous neoplasms, DNA methyltransferase, histone deacetylase

\section{Introduction}

Pancreatic cancer is a highly malignant disease. Early metastasis, local invasion, and resistance to conventional therapy significantly challenge the treatment of this disease. Currently, there are no ideal markers that provide sufficient sensitivity and specificity in the clinic, making it difficult for early diag-nosis. Recently, pathologists raised the concepts that pancreatic intraepithelial neoplasias (PanINs) and intraductal papillary mucinous neoplasms (IPMNs) are precancerous lesions for pancreatic ductal adenocarcinoma (PDAC), providing new targets for studies on the initiation of pancreatic cancer (1-3). Several studies have focused on the molecular biology and histopathological changes associated with PanINs and IPMNs, and demonstrated their importance in the progression of pancreatic cancer (4-8). Therefore, an in-depth study of molecular alterations during the progression from PanINs and IPMNs to PDAC may reveal the pathogenesis of pancreatic cancer.

Epigenetic modifications play an important role during carcinogenesis $(9,10)$. Aberrant methylation, including an overall decrease in DNA methylation and locally increased methylations of $\mathrm{CpG}$ islands, leads to chromosome instability and functional loss of tumor suppressor genes, constituting an early event in tumorigenesis (11-13). Histone acetylation, which modulates the transcription of target genes, is also involved in the formation of various human tumors (14-16). It was reported that these two processes may work together to inhibit transcription of target genes through Methyl-CpG binding proteins (MeCPs), which recruit histone deacetylases (HDACs) to deacetylate histone proteins (17-19). Some studies suggest that DNA methylation functions as an initiator of transcriptional repression, while deacetylation is the executor (20). Moreover, it was reported that DNA methyltransferase-1 (DNMT1) and histone deacetylase-1 (HDAC1), two critical enzymes in epigenetic regulation, repress target gene expression, as suggested by the ability of HDAC1 to bind DNMT1 and to purify methyltransferase activity from nuclear extracts (21).

The expression of DNMT1 or HDAC1 at both the mRNA and protein levels increase in several human tumors (22-26). However, no previous studies, have examined their expression in pancreatic cancer, especially in the precancerous lesions of PanINs and IPMNs. In this study, we applied immunohistochemistry (IHC) to examine the expression of DNMT1 and HDAC1 in a large series of normal, PanINs, IPMNs and 
PDAC samples, and analyzed their significance in the development of pancreatic cancer.

\section{Materials and methods}

All tissue samples for IHC detection of DNMT1 and HDAC1 were acquired from the Department of Pathology, Changhai Hospital (the Second Military Medical University, Shanghai, China): 10 normal pancreatic tissues from autopsy, 15 samples of chronic pancreatitis from 13 males and 2 females (average age 51.9 years), 39 paratumor tissues from patients with PanINs originating from 22 males and 17 females (average age 57.7 years), 48 samples of IPMNs from 25 males and 23 females (average age 58.4 years), and 54 samples of pancreatic cancers from 41 males and 13 females (average age 57.6 years).

All tissues were formalin-fixed, paraffin-embedded, sliced into $4-\mu \mathrm{m}$ sections and examined by H\&E staining. Based on the classification criteria for precursor lesions in the pancreatic ducts (27), the above samples include 20 foci containing normal ductal epithelial cells without an inflammatory background (DE), 30 containing ductal epithelial cells with an inflammatory background (DEI), 48 of PanIN-1A, 103 of PanIN-1B, 99 of PanIN-2, 30 of PanIN-3, 18 of IPMA, 10 of IPMB, 20 of IPMC and 54 PDAC.

The clinicopathological parameters of the 54 patients with pancreatic cancer (PDAC) included age, gender, smoking, alcohol consumption, size of tumor, location of tumor, vessel infiltration, nerve infiltration, tumor differentiation, proliferative activity and TNM staging. Positivity for smoking was defined as at least one cigarette per day for no less than 1 year. Positivity for alcohol consumption was defined as a one-time intake of at least $50 \mathrm{~g}$ of Chinese liquor, $250 \mathrm{ml}$ wine or $500 \mathrm{ml}$ beer, at least once a week for a minimum of 1 year.

IHC was performed on 4 - $\mu$ m-thick serial paraffin sections. Sections were deparaffinized and rehydrated prior to blockage of endogenous peroxidase using 3\% hydrogen peroxide for $5 \mathrm{~min}$. Antigen retrieval was then performed in a microwave oven at $95^{\circ} \mathrm{C}$ for 20 min with slides immersed in antigen retrieval solution $(10 \mathrm{mM}$ sodium citrate buffer, $\mathrm{pH} 6.0$ ) followed by a blocking step with $1 \%$ fetal calf serum for $20 \mathrm{~min}$ at room temperature. After blocking, the sections were incubated at $4{ }^{\circ} \mathrm{C}$ overnight with a primary antibody, followed by incubation with a secondary antibody (goat antirabbit/horseradish peroxidase, R\&D Systems, 1:200 dilution) at room temperature for $30 \mathrm{~min}$. Finally, the sections were developed with DAB substrate for about $5 \mathrm{~min}$, and counterstained with Mayer's hematoxylin. The primary antibodies used in this study included DNMT1 (ab19905, Abcam, 1:200 dilution) and HDAC1 (\#2062, Cell Signaling, 1:400 dilution). PBS was used as a negative control for the primary antibody. Human tonsil tissues were used as positive control for DNMT1 immunoreactivity and human breast cancer tissues for HDAC1 immunoreactivity, as recommended by the manufacturers for the corresponding antibodies.

The IHC data for DNMT1 and HDAC1 were evaluated by two independent observers in a blinded manner. In DE, DEI, PanINs, and IPMNs foci, expression levels for both proteins were calculated as the percentage ratio of the number of ductal epithelial cells with positive staining to the total number of ductal epithelial cells counted. For PDAC foci, since it is difficult to discriminate the ductal and acinar structures, the expression levels were calculated as the percentage of positive stained cells to total number of cells within a high power field $(400 \mathrm{x})$ and at least 5 high power fields were counted for each case.

Comparison of the positivity for DNMT1 and HDAC1 between different groups was done by the Mann-Whitney U or Kruskal-Wallis tests (SPSS software, Version 13.0). Correlations between the expression levels of DNMT1 and HDAC1 and the clinicopathological parameters were analyzed by the $\chi^{2}$ text. Correlation between the expression of DNMT1 and HDAC1 was analyzed by a non-parametic correlation test. The significance of Kaplan-Meier survival curves was calculated by the log-rank test. A p-value of $<0.05$ was considered statistically significant.

\section{Results}

Expression of DNMT1 and HDAC1 in various pancreatic tissues. In all pancreatic tissues examined, the expression of DNMT1 and HDAC1 was only detected in the nucleus and not in the cytoplasm or cell membrane. In normal pancreatic ductal epithelial cells, there were no detectable signals for DNMT1 or HDAC1. Comparison between PanIN and PDAC samples suggested that weak staining of DNMT1 and HDAC1 started to show up in PanIN-1A tissues, with statistically significant increased staining in the order of PanIN-1A $\rightarrow$ PanIN-1B $\rightarrow$ PanIN-2 $\rightarrow$ PanIN3 $\rightarrow$ PDAC $(\mathrm{p}<0.05)$ (Figs. 1 and 2). This is also true for the expression of DNMT1 and HDAC1 between IPMN and PDAC samples, that is, the staining intensities for both proteins increased significantly in the order of DE $\rightarrow$ IPMA $\rightarrow$ IPMB $\rightarrow$ IPMC $\rightarrow$ PDAC $(\mathrm{p}<0.05)$ (Fig. 3). When compared between the same pathological levels of PanIN and IPMN samples, i.e., PanIN-1 compared to IPMA, PanIN-2 to IPMB, and PanIN-3 to IPMC, the expression of both DNMT1 and HDAC1 are significantly higher in PanIN samples than in the corresponding IPMN samples $(\mathrm{p}<0.05)$.

Correlations of DNMT1 and HDAC1 protein expression with clinicopathological variables of PDAC patients. To gain more insight into the clinical significance of DNMT1 and HDAC1 expression in pancreatic cancer, we investigated the association of DNMT1 and/or HDAC1 expression with clinicopathological parameters that reflect the progression and aggressiveness of the tumor. The mean expression level of DNMT1 in PDACs was 59.1\% (95\% confidence interval (CI): $51.3-66.9 \%)$, while that for HDAC1 was $83.1 \%(95 \%$ CI: $78.2-88.1 \%)$. After overall evaluation for the expression of both proteins, we chose the $60 \%$ and $50 \%$ percentiles $(70 \%$ for DNMT1 expression and $90 \%$ for HDAC1 expression) as the cut-off value for high and low level expression of DNMT1 and HDAC1, respectively, since these two values best predict the survival of patients. High levels of DNMT1 expression significantly correlated with nerve infiltration, degree of tumor differentiation and TNM staging $(\mathrm{p}<0.05)$. The high level of HDAC1 expression statistically correlated with the degree of tumor differentiation, proliferative activity 


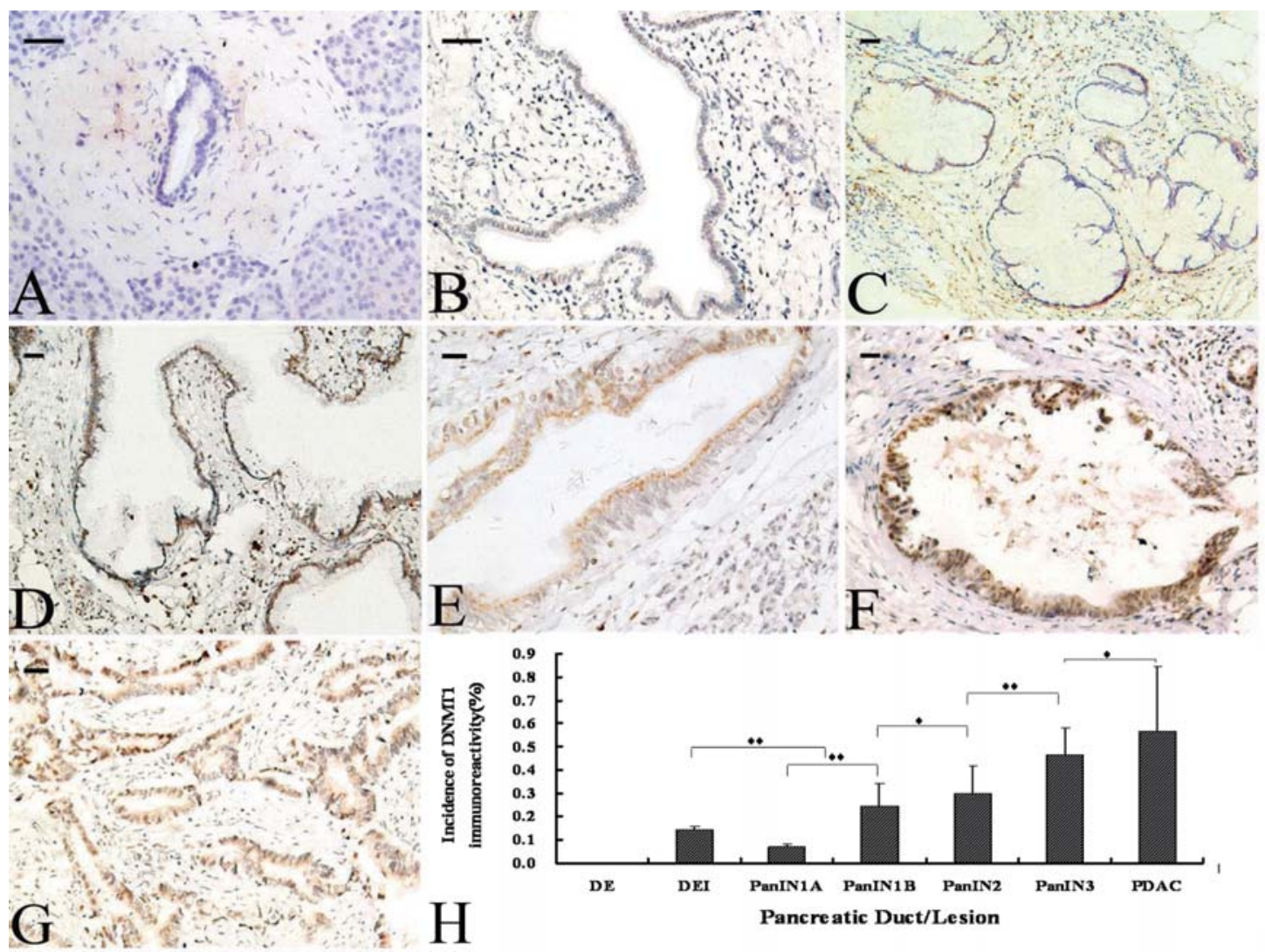

Figure 1. DNMT1 protein immunostaining in pancreatic ductal epithelia with different tissue lesions. (A) DE; (B) DEI; (C) PanIN-1A; (D) PanIN-1B; (E) PanIN-2; (F) PanIN-3; (G) PDAC; (H) *p $<0.01,{ }^{* *} \mathrm{p}<0.001$, bar, $500 \mu \mathrm{m}$.

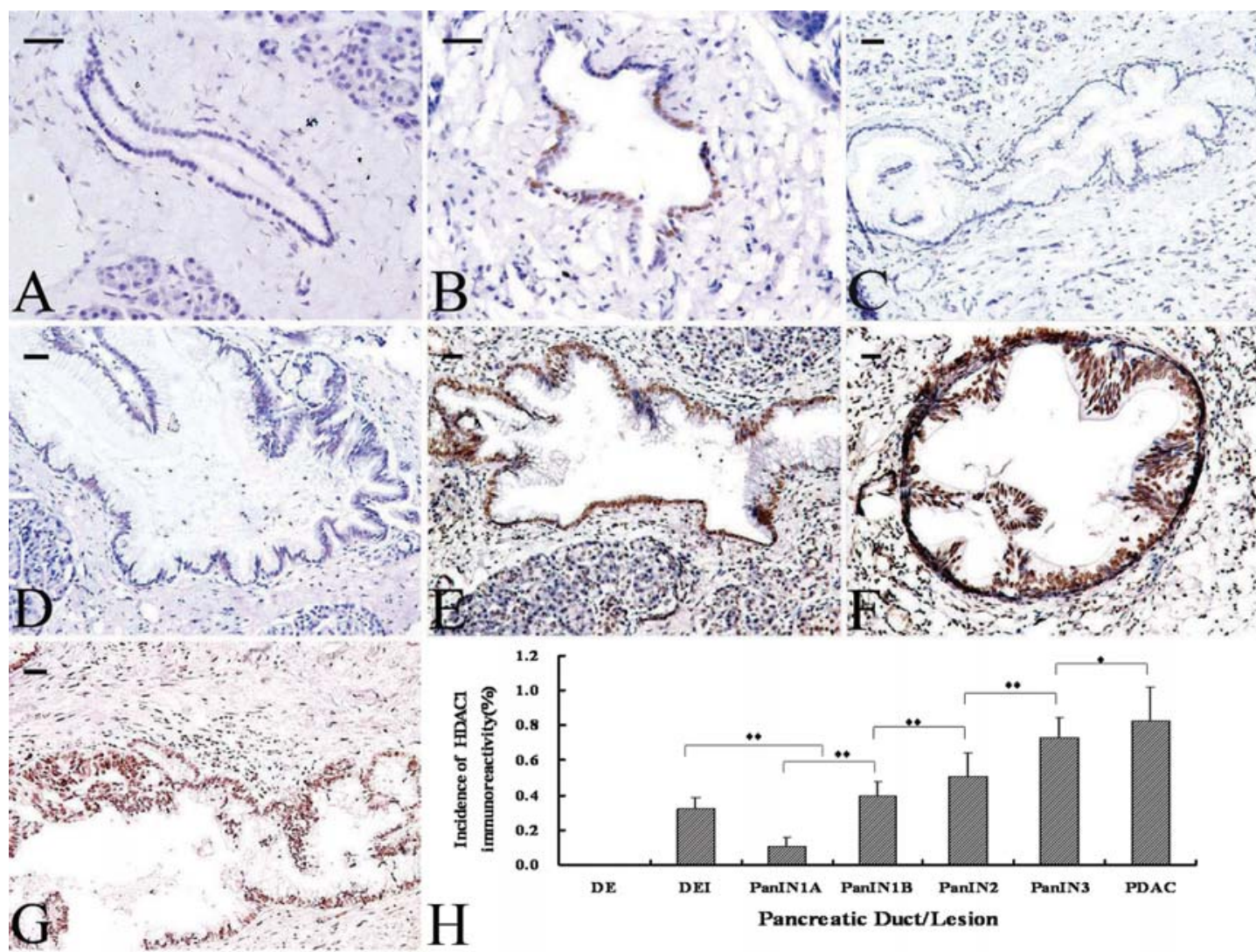

Figure 2. HDAC1 protein immunostaining in pancreatic ductal epithelia with different tissue lesions. (A) DE; (B) DEI; (C) PanIN-1A; (D) PanIN-1B; (E) PanIN-2; (F) PanIN-3; (G) PDAC; (H) ${ }^{*} \mathrm{p}<0.01,{ }^{* *} \mathrm{p}<0.001$, bar, $500 \mu \mathrm{m}$. 


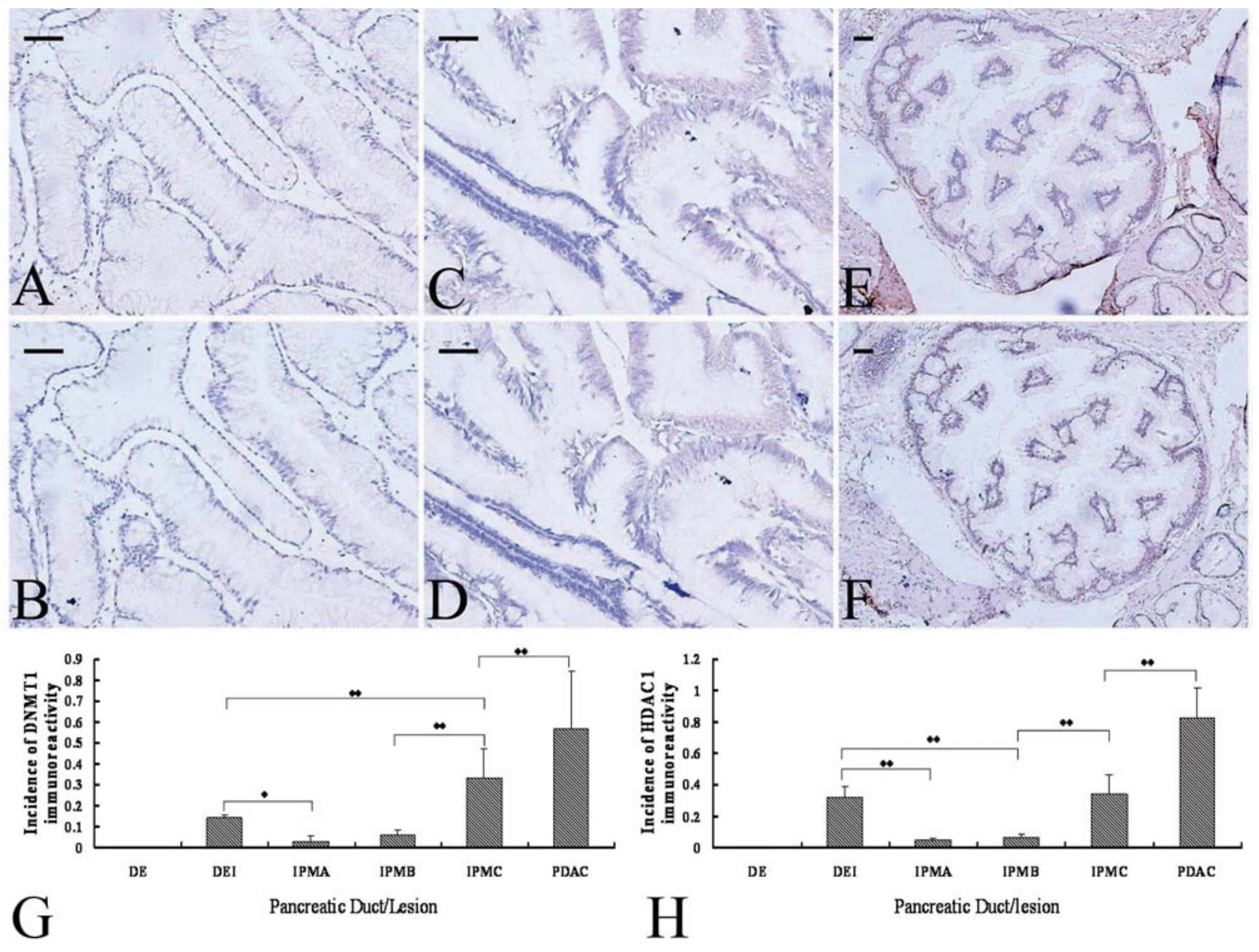

Figure 3. DNMT1 and HDAC1 protein immunostaining in the intraductal components of IPMNs. (A) DNMT1 in IPMA; (B) HDAC1 in IPMA; (C) DNMT1 in IPMB; (D) HDAC1 in IPMB; (E) DNMT1 in IPMC; (F) HDAC1 in IPMC; (G and H) ${ }^{*} \mathrm{p}<0.01,{ }^{* *} \mathrm{p}<0.001$, bar, $500 \mu \mathrm{m}$.

and TNM staging $(\mathrm{p}<0.05)$. No significant correlation was observed between the levels of DNMT1 and HDAC1 protein expression and patient age, gender, smoking, alcohol consumption, tumor size, tumor location, or vessel infiltration (Table I).

Correlations between DNMT1 and HDAC1 expression levels and patient survival. To evaluate the effects of DNMT1 and HDAC1 expression on the prognosis of patients with PDACs, we performed both univariate and multivariate survival analyses. Univariate analysis suggested that alcohol consumption, degree of tumor differentiation, and expression levels of DNMT1 and HDAC1 all significantly correlated with patient survival (Table II). The two-year survival for patients with high expression of DNMT1 was 6\%, significantly lower than that for patients with low expression ( $28 \%, p=0.002)$. Similarly, the two-year survival for patients with high expression of HDAC1 was $5 \%$, significantly lower than that for patients with low expression $(32 \%, \mathrm{p}=0.003)$. For patients with high expression levels of both proteins, the two-year survival was $0 \%$, compared to $6 \%$ in those with only one protein expressed at high levels and $11 \%$ in those with both proteins expressed at low levels $(\mathrm{p}=0.000)$ (Fig. 4).
Multivariate, Cox regression analysis indicated that alcohol consumption, degree of tumor differentiation, TNM staging and expression of DNMT1 and HDAC1 are independent risk factors for predicting prognosis for patients with PDAC. Patients positive for alcohol consumption have a 2.071-fold higher risk of death than those that are negative. The risk for patients with moderately differentiated tumors was 1.965 -fold of those with well differentiated tumors, compared to 9.019-fold for those with poorly differentiated tumors. The risk ratio for patients with TNM stages of III + IV to those with I + II was 3.94. The risk ratio for patients with high levels of expression of DNMT1 to those with low levels was 10.162 and that for HDAC1 expression was 5.286 (Table III).

\section{Discussion}

Accumulating evidence suggests that DNA methylation and histone deacetylation play important roles during carcinogenesis by selectively repressing gene transcription. As a critical enzyme in mammalian cells, DNMT1 is primarily responsible for copying methylation patterns from parental strands to the newly synthesized daughter DNA molecules 
Table I. Correlations of DNMT1 and HDAC1 protein expression with clinicopathological variables $(\mathrm{n}=54)$.

\begin{tabular}{|c|c|c|c|c|c|c|}
\hline \multirow{2}{*}{$\begin{array}{l}\text { Clinicopathological } \\
\text { parameters }\end{array}$} & \multicolumn{2}{|c|}{ DNMT1 } & \multirow[b]{2}{*}{ P-value } & \multicolumn{2}{|c|}{ HDAC1 } & \multirow[b]{2}{*}{$\mathrm{P}$-value } \\
\hline & Low level & High level & & Low level & High level & \\
\hline Age $($ mean $\pm \mathrm{SD})$ & & & 0.314 & & & 0.790 \\
\hline$<60$ & 18 & 7 & & 13 & 12 & \\
\hline$\geq 60$ & 17 & 12 & & 14 & 15 & \\
\hline Gender & & & 0.110 & & & 0.116 \\
\hline Male & 29 & 12 & & 23 & 18 & \\
\hline Female & 6 & 7 & & 4 & 9 & \\
\hline Smoking & & & 0.609 & & & 0.419 \\
\hline Yes & 14 & 9 & & 10 & 13 & \\
\hline No & 21 & 10 & & 17 & 14 & \\
\hline Alcohol consumption & & & 0.656 & & & 0.790 \\
\hline Yes & 17 & 8 & & 13 & 12 & \\
\hline No & 18 & 11 & & 14 & 15 & \\
\hline Nerve infiltration & & & 0.030 & & & 1.000 \\
\hline Positive & 17 & 15 & & 16 & 16 & \\
\hline Negative & 18 & 4 & & 11 & 11 & \\
\hline Vessel infiltration & & & 0.412 & & & 0.522 \\
\hline Positive & 9 & 3 & & 5 & 7 & \\
\hline Negative & 26 & 16 & & 22 & 20 & \\
\hline Location of tumor & & & 0.694 & & & 0.256 \\
\hline Head & 24 & 12 & & 16 & 20 & \\
\hline Body and tail & 11 & 7 & & 11 & 7 & \\
\hline Size of tumor $(\mathrm{cm})$ & & & 0.282 & & & 0.767 \\
\hline$\leq 2$ & 8 & 7 & & 8 & 7 & \\
\hline$>2$ & 27 & 12 & & 19 & 20 & \\
\hline Differentiation & & & 0.000 & & & 0.000 \\
\hline Well & 19 & 0 & & 17 & 2 & \\
\hline Moderate & 14 & 13 & & 10 & 17 & \\
\hline Poor & 2 & 6 & & 0 & 8 & \\
\hline Proliferative activity & & & 0.236 & & & 0.002 \\
\hline High & 18 & 13 & & 10 & 21 & \\
\hline Low & 17 & 6 & & 17 & 6 & \\
\hline TNM staging & & & 0.035 & & & 0.002 \\
\hline $\mathrm{I}+\mathrm{II}$ & 25 & 8 & & 22 & 11 & \\
\hline III + IV & 10 & 11 & & 5 & 16 & \\
\hline
\end{tabular}

(28-31). In tumors, the DNMT1 gene has been implicated as a mutagen for tumor suppressor genes by inducing persistent hypermethylation and subsequent TA base mutations of $\mathrm{CpG}$ islands located within the promoter regions of these genes. Enhanced DNMT1 activity is an initial event in carcinogenesis and constitutes the early phenotypic changes that characterize transformed cells (32). HDAC1, another enzyme involved in transcriptional repression, can remove an acetyl group from the positively charged lysine residues at the N-termini of histone proteins. This post-translational modification adds positive charges to histone proteins and increases their affinity for DNA, which induces a more compact chromatin structure and leads to gene silencing $(14,15,16,33)$. In tumorigenesis, the cooperation between DNMT1 and HDAC1 has been suggested, DNA methylation initiates the process of transcriptional repression, which is then executed by histone deacetylation.

Few studies have reported the function of DNMT1 and HDAC1 in pancreatic cancer. In our current study, we examined the expression of both proteins by IHC in a large 
Table II. Influence of DNMT1 and HDAC1 expression and clinicopathological variables on patient survival (n=54).

\begin{tabular}{|c|c|c|c|c|c|}
\hline & Cases & Events & Mean survival (months) & SE & Log-rank \\
\hline \multicolumn{6}{|l|}{ Gender } \\
\hline Male & 41 & 34 & 11.7 & 1.5 & \\
\hline Female & 13 & 7 & 15.6 & 2.9 & 0.314 \\
\hline \multicolumn{6}{|l|}{ Age } \\
\hline$<60$ & 25 & 19 & 12.9 & 2.0 & \\
\hline$\geq 60$ & 29 & 22 & 12.6 & 1.9 & 0.828 \\
\hline \multicolumn{6}{|l|}{ Smoking } \\
\hline No & 31 & 22 & 14.8 & 1.9 & \\
\hline Yes & 23 & 19 & 9.8 & 1.7 & 0.051 \\
\hline \multicolumn{6}{|c|}{ Alcohol consumption } \\
\hline No & 29 & 20 & 15.5 & 2.0 & \\
\hline Yes & 25 & 21 & 9.3 & 1.5 & 0.023 \\
\hline \multicolumn{6}{|c|}{ Location of tumor } \\
\hline Head & 36 & 27 & 12.8 & 1.7 & \\
\hline Body and tail & 18 & 14 & 12.6 & 2.2 & 0.814 \\
\hline \multicolumn{6}{|l|}{ Size of tumor } \\
\hline$\leq 2$ & 15 & 10 & 13.0 & 3.0 & \\
\hline$>2$ & 39 & 31 & 12.6 & 1.5 & 0.990 \\
\hline \multicolumn{6}{|l|}{ Differentiation } \\
\hline Well & 19 & 13 & 15.8 & 2.5 & \\
\hline Moderate & 27 & 22 & 11.8 & 1.7 & \\
\hline Poor & 8 & 6 & 6.3 & 1.3 & 0.048 \\
\hline \multicolumn{6}{|c|}{ Proliferation activity } \\
\hline Low & 23 & 17 & 14.3 & 2.2 & \\
\hline High & 31 & 24 & 11.3 & 1.6 & 0.307 \\
\hline \multicolumn{6}{|c|}{ Nerve infiltration } \\
\hline No & 22 & 16 & 13.2 & 2.3 & \\
\hline Yes & 32 & 25 & 12.3 & 1.6 & 0.852 \\
\hline \multicolumn{6}{|c|}{ Vessel infiltration } \\
\hline No & 42 & 32 & 13.1 & 1.6 & \\
\hline Yes & 12 & 9 & 11.4 & 2.6 & 0.704 \\
\hline \multicolumn{6}{|l|}{ TNM } \\
\hline $\mathrm{I}+\mathrm{II}$ & 33 & 23 & 16.2 & 1.8 & \\
\hline III + IV & 21 & 18 & 6.5 & 0.9 & 0.000 \\
\hline \multicolumn{6}{|l|}{ DNMT1 } \\
\hline Low & 35 & 25 & 15.2 & 1.7 & \\
\hline High & 19 & 16 & 7.9 & 1.6 & 0.002 \\
\hline \multicolumn{6}{|l|}{ HDAC1 } \\
\hline Low & 27 & 18 & 16.4 & 2.1 & \\
\hline High & 27 & 23 & 8.7 & 1.3 & 0.003 \\
\hline \multicolumn{6}{|c|}{ DNMT1 and HDAC1 } \\
\hline Both low & 20 & 12 & 18.3 & 2.4 & \\
\hline Either high & 21 & 18 & 10.7 & 1.6 & \\
\hline Both high & 13 & 11 & 6.2 & 1.0 & 0.000 \\
\hline
\end{tabular}

number of clinical samples representing different stages of pancreatic cancer development. Our study showed that expression of DNMT1 and HDAC1 increased with the development of pancreatic cancer from normal tissue to precan- 
$\mathbf{A}$

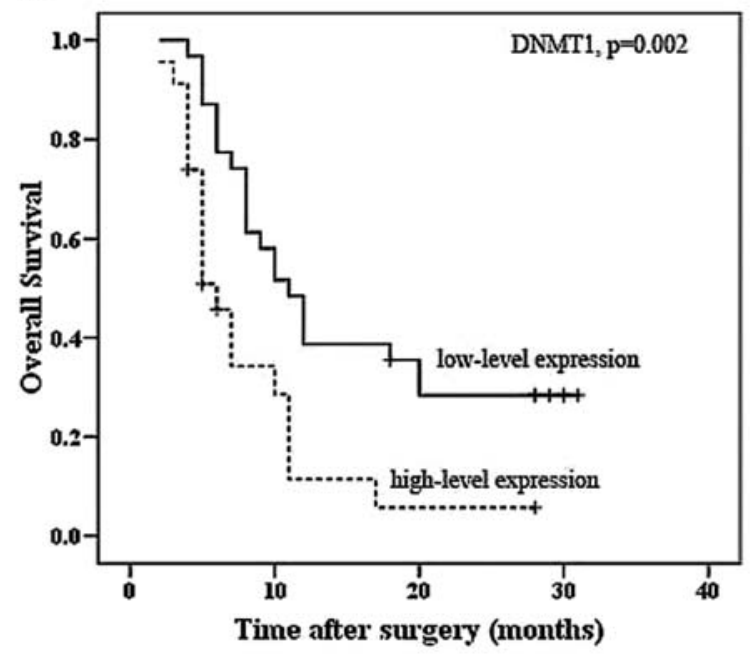

B

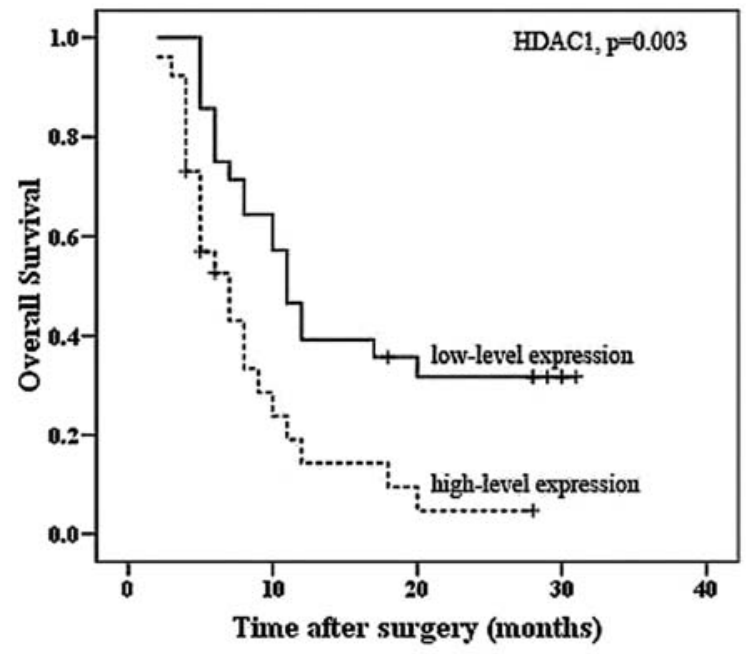

C

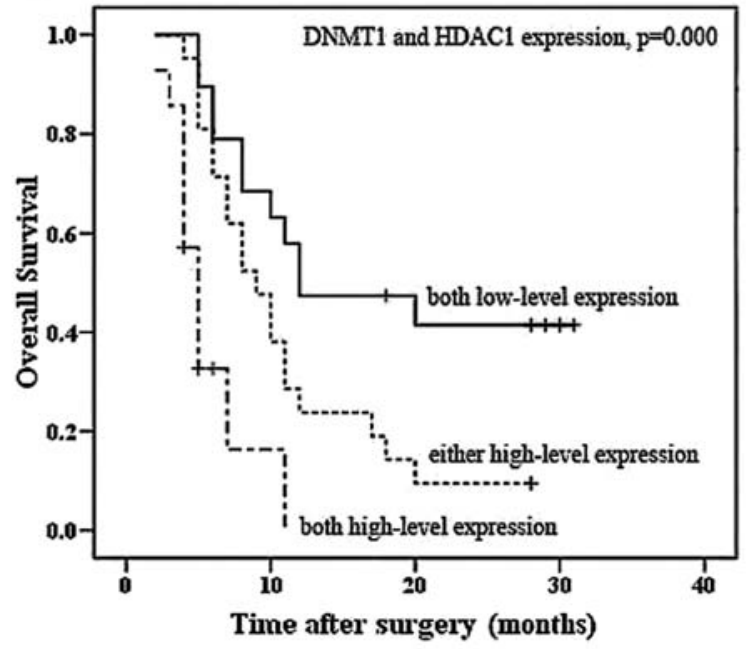

Figure 4. Kaplan-Meier survival curves based on DNMT1 and HDAC1 protein expression levels. Overall survival according to the protein expression of DNMT1 (A), HDAC1 (B), and both (C). P-value was calculated with the log-rank test.

cerous lesions, PanIN and IPMN, and to cancer PDAC. Positive immunostaining for both proteins are detectable as early as in PanIN-1A and IPMA lesions, suggesting that
Table III. Cox regression analysis under inclusion of clinicopathological factors and DNMT1 and HDAC1 expression $(n=54)$.

\begin{tabular}{lcc}
\hline & \multicolumn{2}{c}{ Overall survival } \\
\cline { 2 - 3 } & HR $(95 \%$ CI $)$ & P-value \\
\hline Alcohol consumption & 1 & 0.040 \\
$\quad$ No & & \\
Yes & $2.071(1.033-4.152)$ & 0.002 \\
TNM & 1 & \\
I + II & $3.94(1.685-9.211)$ & 0.026 \\
III + IV & 1 & 0.212 \\
Differentiation & $1.965(0.680-5.679)$ \\
Well & $9.019(1.639-49.621)$ & 0.011 \\
Moderate & & 0.000 \\
Poor & 1 & \\
DNMT1 & & \\
Low & & \\
High & $10.162(3.424-30.156)$ & \\
HCAC1 & & \\
Low & & \\
High & $5.286(1.905-14.663)$ \\
\hline
\end{tabular}

HR, risk ratio; 95\% CI, 95\% confidence interval.

increased expression of DNMT1 and HDAC1 was an early molecular event and more importantly, may drive the progression to PDAC. When analyzing the expression pattern between DNMT1 and HDAC1 in all samples, we found a significant and linear correlation between the expression of these two proteins $(\mathrm{r}=0.970, \mathrm{p}<0.001)$, indicating that these two enzymes coordinately regulate epigenetic modification during pancreatic carcinogenesis. In addition, when we compared the expression levels of DNMT1 and HDAC1 proteins between PanIN and IPMN lesions, two precancerous lesions with distinct molecular, pathological and clinical features (34-36), we found that expression of both proteins in PanIN lesions was always higher than in the corresponding stages of IPMN lesions, implying IPMN as a relatively indolent and benign lesion compared with PanIN.

To analyze the influence of inflammation on the expression of DNMT1 and HDAC1, we compared their expression levels in DEI samples with those in other lesion groups, and found that the expression levels for both proteins in DEI lesions were between those for PanIN-1 and PanIN-2 lesions (Figs. 1 and 2). Consistent with a previous study (26), we noted that persistent inflammation influences epigenetic regulation, as demonstrated by the up-regulation of DNMT1 and HDAC1 in our study. This observation supports the idea that chronic pancreatitis could be a risk factor for pancreatic cancer.

The enhanced expression of both DNMT1 and HDAC1 in the development of pancreatic cancer suggest they may function synergistically at the molecular level. The correlation 
analysis between the expression levels of DNMT1 and HDAC1 protein and the clinicopathological parameters revealed that there was a statistically significant correlation between high levels of DNMT1 expression and nerve infiltration, degree of tumor differentiation and TNM staging; the high level of HDAC1 expression correlated significantly with degree of tumor differentiation, proliferative activity and TNM staging. No significant correlations were observed between the expression of DNMT1 and HDAC1 and patient age, gender and other clinicopathological parameters. Some earlier studies suggested that increased expression of DNMT1 associated with the proliferative activity of tumor cells, and DNMT1 can be loaded onto the replication fork via PCNA, a replication factor $(37,38)$. In this study, we did not find a significant correlation between DNMT1 expression and proliferative activity, as indicated by the proliferative labeling results of Ki67 and PCNA. Recently, several studies identified a role for environmental factors in regulating epigenetic modifications (39-42). Our analysis of the correlation between patient life styles, such as smoking and alcohol consumption, and the expression of DNMT1 and HDAC1, showed no significance.

By the Kaplan-Meier survival analysis, we observed a significant difference in patient survival between groups with high and low DNMT1 or HDAC1 expression, with the high level expression group having a shorter survival time. This result is consistent with a previous study that found a significant difference between survival between the groups with high and low DNMT1 protein expression (26). Our results imply that DNMT1 and HDAC1 could be two useful markers in predicting the prognosis of patients with PDAC.

In conclusion, our study adds new evidence supporting the role of DNMT1 and HDAC1 in driving the progression of PDAC. Importantly, they are significantly related to the clinical outcomes of patients with pancreatic cancer, and may serve as valuable prognostic markers for PDAC. Although further study is warranted to determine the precise roles of DNMT1 and HDAC1 during pancreatic tumorigenesis, the results of the present study imply that DNMT1 and HDAC1 may be potential therapeutic targets for cancer therapy.

\section{Acknowledgements}

We thank Jian-Ming Zheng and Li Gao for assistance in performing the immunohistochemistry staining and evaluation of histopathological grades of the PanINs, IPMNs, and other lesions as well as immunostaining intensity.

\section{References}

1. Hruban RH, Adsay NV, Albores-Saavedra J, et al: Pancreatic intraepithelial neoplasia: a new nomenclature and classification system for pancreatic duct lesions. Am J Surg Pathol 25: 579-586, 2001

2. Takaori K, Hruban RH, Maitra A and Tanigawa N: Pancreatic intraepithelial neoplasia. Pancreas 28: 257-262, 2004.

3. Biankin AV, Kench JG, Biankin SA, et al: Pancreatic intraepithelial neoplasia in association with intraductal papillary mucinous neoplasms of the pancreas: implications for disease progression and recurrence. Am J Surg Pathol 28: 1184-1192, 2004.

4. Hruban RH, Goggins M, Parsons J and Kern SE: Progression model for pancreatic cancer. Clin Cancer Res 6: 2969-2972, 2000 .
5. Cowgill SM and Muscarella P: The genetics of pancreatic cancer. Am J Surg 186: 279-286, 2003.

6. Biankin AV, Kench JG, Dijkman FP, Biankin SA and Henshall SM: Molecular pathogenesis of precursor lesions of pancreatic ductal adenocarcinoma. Pathology 35: 14-24, 2003.

7. Segara D, Biankin AV, Kench JG, et al: Expression of $\mathrm{HOXB} 2$, a retinoic acid signaling target in pancreatic cancer and pancreatic intraepithelial neoplasia. Clin Cancer Res 11: 3587-3596, 2005.

8. Hiraoka N, Onozato K, Kosuge T and Hirohashi S: Prevalence of $\mathrm{FOXP}^{+}$regulatory $\mathrm{T}$ cells increases during the progression of pancreatic ductal adenocarcinoma and its premalignant lesions. Clin Cancer Res 12: 5423-5434, 2006.

9. Sato N, Matsubayashi H, Abe T, Fukushima N and Goggins M: Epigenetic down-regulation of CDKN1C/p57KIP2 in pancreatic ductal neoplasms identified by gene expression profiling. Clin Cancer Res 11: 4681-4688, 2005.

10. Kurdistani SK: Histone modifications as markers of cancer prognosis: a cellular view. Br J Cancer 97: 1-5, 2007.

11. Kanai Y, Ushijima S, Tsuda H, Sakamoto M, Sugimura T and Hirohashi S: Aberrant DNA methylation on chromosome 16 is an early event in hepatocarcinogenesis. Cancer Sci 87: 1210-1217, 1996.

12. Kanai Y, Hui AM, Sun L, Ushijima S, Sakamoto M, Tsuda H and Hirohashi S: DNA hypermethylation at the D17S5 locus and reduced HIC-1 mRNA expression are associated with hepatocarcinogenesis. Hepatology 29: 703-709, 1999.

13. Kondo Y, Kanai Y, Sakamoto M, Mizokami M, Ueda R and Hirohashi S: Genetic instability and aberrant DNA methylation in chronic hepatitis and cirrhosis: a comprehensive study of loss of heterogeneity and microsatellite instability at 39 loci and DNA hypermethylation on $8 \mathrm{CpG}$ islands in microdissected specimens from patients with hepatocellular carcinoma. Hepatology 32: 970-979, 2000.

14. Grunstein M: Histone acetylation in chromatin structure and transcription. Nature 389: 349-352, 1997.

15. De Ruijter AJ, van Gennip AH, Caron HN, Kemp S and van Kuilenburg AB: Histone deacetylases (HDACs): characterization of the classical HDAC family. Biochem J 370: 737-749, 2003

16. McGraw S, Robert C, Massicotte L and Sirard MA: Quantification of histone acetyltransferase and histone deacetylase transcripts during early bovine embryo development. Biol Reprod 68: 383-389, 2003

17. Jones PL, Veenstra GJ, Wade PA, et al: Methylated DNA and MeCP2 recruit histone deacetylase to repress transcription. Nat Genet 19: 187-191, 1998.

18. Nan X, Ng HH, Johnson CA, Laherty CD, Turner BM, Eisenman RN and Bird A: Transcriptional repression by themethyl-CpG-binding protein MeCP2 involves a histone deacetylase complex. Nature 393: 386-389, 1998.

19. Fuks F, Hurd PJ, Wolf D, Nan X, Bird AP and Kouzarides T: The methyl-CpG-binding protein MeCP2 links DNA methylation to histone methylation. J Biol Chem 278: 4035-4040, 2003.

20. Geiman TM and Robertson KD: Chromatin remodeling, histone modifications, and DNA methylation - how does it all fit together? J Cell Biochem 87: 117-125, 2002.

21. Fuks F, Burgers WA, Brehm A, Hughes-Davies L and Kouzarides T: DNA methyltransferase Dnmt1 associates with histone deacetylase activity. Nat Genet 24: 88-91, 2000.

22. Melki JR, Warnecke P, Vincent PC and Clark SJ: Increased DNA methyltransferase expression in leukaemia. Leukemia 12: 311-316, 1998

23. Saito Y, Kanai Y, Sakamoto M, Saito H, Ishii H and Hirohashi S: Expression of mRNA for DNA methyltransferases and methyl$\mathrm{CpG}$-binding proteins and DNA methylation statuses on $\mathrm{CpG}$ islands and pericentromeric satellite regions during human hepatocarcinogenesis. Hepatology 33: 561-568, 2001.

24. Omisanjo OA, Biermann K, Hartmann S, et al: DNMT1 and HDAC1 gene expression in impaired spermatogenesis and testicular cancer. Histochem Cell Biol 127: 175-181, 2007.

25. Sasaki H, Moriyama S, Nakashima Y, et al: Histone deacetylase 1 mRNA expression in lung cancer. Lung Cancer 46: 171-178, 2004.

26. Peng DF, Kanai Y, Sawada M, Ushijima S, Hiraoka N, Kosuge T and Hirohashi S: Increased DNA methyltransferase 1 (DNMT1) protein expression in precancerous conditions and ductal carcinomas of the pancreas. Cancer Sci 96: 403-408, 2005. 
27. Hruban RH, Takaori K, Klimstra DS, et al: An illustrated consensus on the classification of pancreatic intraepithelial neoplasia (PanIN) and intraducatal papillary mucinous neoplasms (IPMNs). Am J Surg Pathol 28: 977-987, 2004.

28. Jones PA: DNA methylation and cancer. Oncogene 21: 5358-5360, 2002.

29. Herman JG and Baylin SB: Gene silence in cancer in association with promoter methylation. N Engl J Med 349: 2042-2054, 2003.

30. Szyf M: DNA methylation patterns: an additional level of information? Biochem Cell Biol 69: 764-767, 1991.

31. Leonhardt H, Page AW, Weier HU and Bestor TH: A targeting sequence directs DNA methyltransferase to sites of DNA replication in mammalian nuclei. Cell 71: 865-873, 1992.

32. Qayum I and Ashraf M: DNA methyltransferase 1 (DNMT1) gene activity in human lymphomas correlates with aberrant p53 gene expression. J Ayub Med Coll Abbottabad 18: 1-6, 2006.

33. Spencer VA and Davie JR: Role of covalent modifications of histones in regulating gene expression. Gene 240: 1-12, 1999.

34. Adsay NV, Merati K, Andea A, et al: The dichotomy in the preinvasive neoplasia to invasive carcinoma sequence in the pancreas: differential expression of MUC1 and MUC2 supports the existence of two separate pathways of carcinogenesis. Mod Pathol 15: 1087-1095, 2002.
35. Moriya T, Kimura W, Semba S, et al: Biological similarities and differences between pancreatic intraepithelial neoplasias and intraductal papillary mucinous neoplasms. Int J Gastrointest Cancer 35: 111-119, 2005.

36. Wang Y, Gao J, Li Z, Jin Z, Gong Y and Man X: Diagnostic value of mucins (MUC1, MUC2 and MUC5AC) expression profile in endoscopic ultrasound-guided fine-needle aspiration specimens of the pancreas. Int J Cancer 121: 2716-2722, 2007.

37. Chuang LS, Ian HI, Koh TW, Ng HH, Xu G and Li BF: Human DNA-(Cytosine-5) methyltransferase-PCNA complex as a target for p21WAF1. Science 277: 1996-2000, 1997.

38. Esteve PO, Chin HG, Smallwood A, et al: Direct interaction between DNMT1 and G9a coordinates DNA and histone methylation during replication. Genes Dev 20: 3089-3103, 2006.

39. Lin R, Hsu H, Chang J, Chen C, Chen J and Wang Y: Alteration of DNA methyltransferases contributes to $5^{\prime} \mathrm{CpG}$ methylation and poor prognosis in lung cancer. Lung Cancer 55: 205-213, 2007.

40. Cooney CA, Dave AA and Wolff GL: Maternal methyl supplements in mice affect epigenetic variation and DNA methylation of offspring. J Nutr 132: S2393-S2400, 2002.

41. Wolff GL: Regulation of yellow pigment formation in mice: a historical perspective. Pigment Cell Res 16: 2-15, 2003.

42. Simmons RA: Developmental origins of beta-cell failure in type 2 diabetes: the role of epigenetic mechanisms. Pediatr Res 61: R64-R67, 2007. 